\section{Commentary: All that glitters isn't gold: Defining low-risk lesions in early-stage esophageal adenocarcinoma}

\author{
Joanna Sesti, MD, and Subroto Paul, MD, MPH
}

In this issue of the Journal, Shihag and colleagues ${ }^{1}$ identify risk factors for recurrence among patients with completely resected $\mathrm{T} 1$ esophageal adenocarcinoma. The main impetus for this analysis is the paradigm shift of treatment for early-stage esophageal adenocarcinoma away from radical esophagectomy and toward organsparing techniques, such as endoscopic mucosal resection (EMR) and endoscopic submucosal dissection (ESD). The promise of treating esophageal cancer with endoscopy certainly has the promise of alchemy and has been supported by several retrospective institutional series demonstrating comparable overall survival between EMR and esophagectomy as long as a negative margin is achieved. ${ }^{2,3}$ Unfortunately, as the expression goes, all that glitters is not gold, and we must question whether EMR and ESD is the $24 \mathrm{~K}$ magic bullet we seek for early-stage esophageal adenocarcinoma.

The analysis showed an overall survival of $68.6 \%$ at 5 years and a median interval to recurrence of 2.0 years. Most recurrences were distant, with a cumulative incidence of recurrence (CIR) of $11 \%$ for distant recurrence but only $4 \%$ for locoregional recurrence. In multivariable analysis, only pathologic N1 disease and tumor length were significant risk factors for recurrence.

Although useful, this information doesn't allow us to determine which patients may be better suited for endoscopic techniques versus esophagectomy. To that end, the team constructed a final multivariable model using

\footnotetext{
From Thoracic Surgical Services, RWJBarnabas Health, West Orange, NJ. Disclosures: The authors reported no conflicts of interest.

The Journal policy requires editors and reviewers to disclose conflicts of interest and to decline handling or reviewing manuscripts for which they may have a conflict of interest. The editors and reviewers of this article have no conflicts of interest.

Received for publication Nov 27, 2020; revisions received Nov 27, 2020; accepted for publication Nov 30, 2020; available ahead of print Dec 5, 2020.

Address for reprints: Subroto Paul, MD, MPH, Rutgers New Jersey Medical School, RWJBarnabas Health, 101 Old Short Hills Rd, Ste 302, West Orange, NJ 07052 (E-mail: Subroto.Paul@rwjbh.org).

J Thorac Cardiovasc Surg 2021;162:1280-1

$0022-5223 / \$ 36.00$

Copyright (c) 2020 by The American Association for Thoracic Surgery

https://doi.org/10.1016/j.jtcvs.2020.11.144
}

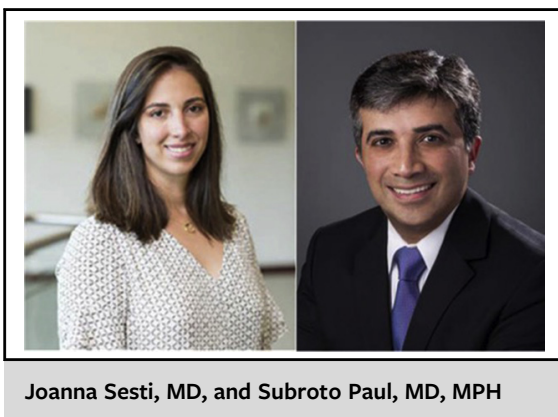

CENTRAL MESSAGE

Among patients with $\mathrm{T}_{1}$ esophageal adenocarcinoma, those with tumors $<2 \mathrm{~cm}$ in length without lymphovascular invasion have a low risk of recurrence.

only clinicopathologic variables that may be known preoperatively. In this model, only lymphovascular invasion (LVI) and tumor length were significant. Finally, using this information, they defined a low-risk group and a high-risk group. They found a significantly higher CIR and risk of death in the high-risk group, validating their stratification.

The authors conclude that patients without LVI and tumor length $<2 \mathrm{~cm}$ have the lowest risk of recurrence $(<10 \%)$ and a 5 -year survival of $75 \%$, which may identify them as good candidates for endoscopic resection. In contrast, patients deemed high-risk (LVI or with tumors $>3 \mathrm{~cm}$ ) have a $>10 \%$ risk of recurrence and may be better treated with esophagectomy.

In the end, it appears that the optimal treatment for $\mathrm{T} 1$ esophageal adenocarcinoma remains to be determined. Although endoscopic techniques shine and glitter as less morbid yet equally effective treatment options for patients with early-stage esophageal cancer, it may be necessary to parcel these patients based on risk of recurrence to determine who will derive the maximal oncologic benefit. Furthermore, with the rate of distant recurrence double that of locoregional recurrence, it remains to be seen if the decision will remain between two surgical techniques or, as we have witnessed in lung cancer, genetically targeted systemic therapies will evolve into the ultimate $24 \mathrm{~K}$ magic bullet.

\section{References}

1. Sihag S, Torre SDL, Hsu M, Nobel T, Tan KS, Gerdes H, et al. Defining low-risk lesions in early-stage esophageal adenocarcinoma. J Thorac Cardiovasc Surg. 2021;162:1272-9. 
2. Ramay FH, Vareedayah AA, Visrodia K, Iyer PG, Wang KK, Eluri S, et al. What constitutes optimal management of T1N0 esophageal adenocarcinoma? Ann Surg Oncol. 2019;26:714-31.
3. Marino KA, Sullivan JL, Weksler B. Esophagectomy versus endoscopic resection for patients with early-stage esophageal adenocarcinoma: a national cancer database propensity-matched study. J Thorac Cardiovasc Surg. 2018;155:2211-8.
See Article page 1272.

\section{Commentary: Risk stratification for superficial esophageal adenocarcinoma: Reducing risk in a risky business!}

\author{
Saurav Adhikari, MD, and Siva Raja, MD, PhD, FACS
}

Organ preservation in the treatment of early esophageal cancer is an accepted paradigm. These techniques are appropriate for carefully selected patients who have earlystage and less aggressive tumors or as a mitigating measure. ${ }^{1}$ Unlike some other malignancies for which the aggressiveness of the cancer, such as lung cancer, can span the spectrum of indolent to aggressive, esophageal adenocarcinoma mostly has aggressive and very aggressive biology. As such, only the earliest of cancers have a low enough risk of metastatic spread at the time of diagnosis that they can be treated with local therapies. However, the identification of this "low-risk" population has remained elusive.

In their article, Sihag and colleagues ${ }^{2}$ have attempted to define criteria associated with a low risk of cancer recurrence in pT1 esophageal adenocarcinoma. In the univariable analysis, they identified the usual suspects for increased risk, such as submucosal invasion, poor differentiation, nodal disease, increased tumor length, and multicentricity. However, using a multivariable model, the authors have demonstrated only increased tumor length and nodal disease to be independent risk factors for recurrence. More interestingly, they set out to identify cancers

\footnotetext{
From the Department of Thoracic and Cardiovascular Surgery, Heart and Vascular Institute, Cleveland Clinic, Cleveland, Ohio.

Disclosures: The authors reported no conflicts of interest.

The Journal policy requires editors and reviewers to disclose conflicts of interest and to decline handling or reviewing manuscripts for which they may have a conflict of interest. The editors and reviewers of this article have no conflicts of interest.

Received for publication Nov 20, 2020; revisions received Nov 20, 2020; accepted for publication Nov 23, 2020; available ahead of print Dec 3, 2020.

Address for reprints: Siva Raja, MD, PhD, FACS, Department of Thoracic and Cardiovascular Surgery, Heart and Vascular Institute, Cleveland Clinic Foundation, J41, 9500 Euclid Ave, Cleveland, OH 44195 (E-mail: rajas@ccf.org).

J Thorac Cardiovasc Surg 2021;162:1281-2

$0022-5223 / \$ 36.00$

Copyright (c) 2020 by The American Association for Thoracic Surgery

https://doi.org/10.1016/j.jtcvs.2020.11.130
}

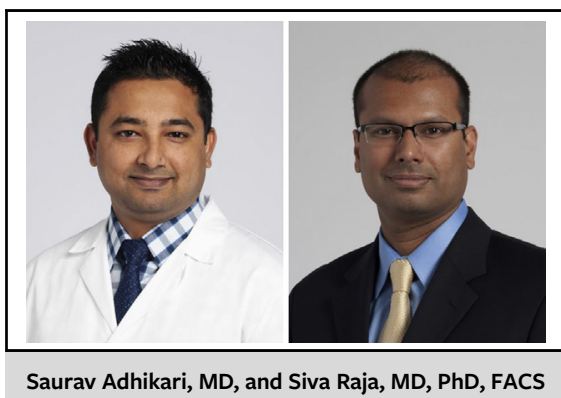

CENTRAL MESSAGE

Early-stage esophageal cancer

may be amenable to endoscopic

therapy, but requires careful

assessment of cancer

characteristics.

with less than $10 \%$ chance of recurrence on the basis of only preoperative clinicopathologic features. In doing so, they noted that patients with tumors less than $2 \mathrm{~cm}$ without lymphovascular invasion had a low rate of recurrence.

It is important to note that the patient population identified in the study includes those with completely resected cancers in whom recurrence status was retrospectively explored. Given that they have had a reasonable nodal dissection, occult metastatic disease (be it in regional lymph nodes or in transit within the esophageal lymphatics) may have been surgically removed in clinically node-negative patients. It is difficult to determine how these results would translate to recurrence in patients after endoscopic resection only in whom a lymphadenectomy had not been performed. Additionally, the authors only found N1 disease to be significant but not depth of invasion or poor differentiation to be a risk factor for recurrence. Although curious at first glance, it is likely because their study population was small, and these, at some level, are related variables.

This original work ${ }^{2}$ adds to our current knowledge base about tumors that are low risk, high risk, and everything in between. Mucosal tumors without poor differentiation or lymphovascular invasion are clearly suited to organ- 